\title{
Le voyage implicite, en compagnie de Haudricourt et de Leroi-Gourhan
}

\section{Catherine Simon}

\section{(2) OpenEdition}

1 Journals

\section{Édition électronique}

URL : http://journals.openedition.org/leportique/3542

DOI : $10.4000 /$ leportique.3542

ISSN : $1777-5280$

\section{Éditeur}

Association "Les Amis du Portique"

\section{Édition imprimée}

Date de publication : 1 septembre 2019

Pagination : 83-99

ISBN : 978-2-916332-40-6

ISSN : 1283-8594

\section{Référence électronique}

Catherine Simon, "Le voyage implicite, en compagnie de Haudricourt et de Leroi-Gourhan », Le

Portique [En ligne], 43-44 | 2019, document 7, mis en ligne le 10 février 2020, consulté le 26 mars 2021.

URL : http://journals.openedition.org/leportique/3542 ; DOI : https://doi.org/10.4000/leportique.3542

Ce document a été généré automatiquement le 26 mars 2021.

Tous droits réservés 


\title{
Le voyage implicite, en compagnie de Haudricourt et de Leroi-Gourhan
}

\author{
Catherine Simon
}

1 Je voudrais pallier par le récit d'un voyage intérieur renvoyant parfois à des souvenirs plus personnels l'absence dans ce numéro d'une étude qui aurait comparé les voyages au Japon de Claude Lévi-Strauss (1908- 2009), d'André Leroi-Gourhan (1911-1986) et d'André-Georges Haudricourt (1911-1996).

2 L'analyse précise de ce qui rapproche et de ce qui sépare les voyages de ces trois anthropologues est un travail ambitieux qu'il restera à faire. Après l'étude magistrale que nous donne Philippe Choulet des écrits de Claude Lévi-Strauss ${ }^{1}$, mon récit met plus modestement en perspective les « voyages des deux André » à partir d'une ou deux impressions de touriste au Japon.

3 André Leroi-Gourhan réside au Japon de mars 1937 à mai 1939 grâce à une dotation du gouvernement japonais qui, pour la première fois, accueille des étudiants étrangers. Son séjour se situe au tout début de sa carrière, juste avant la seconde guerre mondiale, il a vingt-six ans. André-Georges Haudricourt, quant à lui, est simplement l'invité d'un professeur de linguistique de l'université de Tokyo qui a suivi ses cours à Paris. Il reste trois semaines au Japon en octobre 1978. Il a 67 ans, le gros de sa carrière est pour ainsi dire derrière lui.

4 Leurs voyages respectifs au Japon sont connus. Le récit biographique André LeroiGourhan. Une vie (1911-1986) par Philippe Soulier paru en 2018 (éditions CNRS) y consacre le chapitre 2 « Le Japon (mars 1937- mai 1939) ». Par ailleurs Pages oubliées sur le Japon, recueil posthume réuni grâce au soutien de Arlette Leroi-Gourhan (qui l'a accompagné dans un premier temps au Japon) paraît en 2004 et un numéro de Techniques et Culture intitulé « Geste et matière. André Leroi-Gourhan, découvertes japonaises » paraît en 2011.

5 Un article de Tadahiko L. A. Shintani intitulé « Dans l'intimité du professeur A.-G. Haudricourt - son voyage au Japon », paraît dans la revue Le Portique $n^{\circ} 27$, un numéro exclusivement dédié à « André-Georges Haudricourt. La matière du monde » en 2011. 
6 Les travaux et les écrits de ces deux auteurs ont été l'objet d'un intérêt renouvelé, et notablement après leur mort. Plus que des hommages, ce sont des remises au jour de leur esprit d'ouverture et de créativité qu'ont permises les publications tardives de correspondances, les rééditions d'articles, la tenue de colloques avec ceux qui les ont côtoyés et la réalisation d'ouvrages collectifs. Ce travail de visibilité a sans doute aussi contribué à régénérer des pans entiers de l'anthropologie, notamment dans nos manières de penser les rapports à notre environnement et aux autres espèces².

7 En ce qui me concerne, j'ai fait le voyage du Japon au printemps 2018 (du 27 avril au 17 mai) de façon un peu contingente. J'y ai atterri sans réelle préparation. J'ai eu une lecture flottante du récit de Didier Doumergue, Tokyo Script, je ne voulais pas que cette lecture détermine mes impressions, que je me mette à tout voir avec son regard. Je n'attendais rien du Japon, il devait répondre uniquement, pensais- je, à un besoin d'évasion. Cependant l'expérience pratique du voyage a immédiatement réactivé des préoccupations liées à des activités de recherche en ethnologie menées par le passé. Elles m'ont conduite à comparer voyage touristique et voyage à des fins ethnographiques à partir du point précis de la rencontre avec l'autre. C'est ce point que je tente d'investir dans les voyages d'Haudricourt et de Leroi-Gourhan, la place que prend dans leur travail la curiosité pour l'autre indissociable du sentiment d'étrangeté.

8 Michel Naepels encadre un séminaire à l'EHESS intitulé "Questions d'éthique ethnographique »; il propose d'aborder les questions éthiques qui se posent concrètement au cours d'une enquête ethnographique, d'investir les registres de mobilisation de la subjectivité et du corps de l'enquêteur comme celui de ses interlocuteurs et de formuler les questions qui en découlent. Naepels réfléchit aux conditions de la possibilité de l'enquête. Il m'aide à me représenter le malaise de l'enquête ethnographique ${ }^{3}$, souvent refoulé ou nié : une difficulté à trouver la juste place de celui qui interroge, qui collecte des informations sur des populations ou des cultures autres. Le malaise qui naît de la situation ethnographique serait partie prenante du travail en train de se faire. Artefact, il ne pourrait être contourné, et devrait être associé au résultat de l'enquête.

9 La référence au Japon, son association à Leroi-Gourhan sonnent comme une évidence, c'est ce que je ne manque de relever dans le numéro de la revue Techniques et Culture de l'année 2011 : « Avec l'expérience leroi-gourhanienne du Japon à la fin des années 1930, nous retrouvons en genèse, les idées d'esthétique fonctionnelle, de tendance et de degrés du fait, mais aussi les méthodes d'enregistrement et de traitement des données qu'il développera dans Évolution et Techniques (1943-1945) ou plus tard dans Préhistoire de l'art occidental (1965) $»^{4}$.

10 Leroi-Gourhan confie bien après son retour : «Mais décrire le Japon c'est difficile. (...) Et si j'ai craint de dire des banalités, c'est qu'à dire en français les réalités japonaises on les vide beaucoup de leur substance. Cet écart entre ce que l'on vit et ce que l'on peut dire se constate d'ailleurs en toute occasion ». Propos rapportés par Jean-François Lesbre dans « le mot de l'éditeur » de Pages oubliés sur le Japon.

11 Leroi-Gourhan se souvient, quelques années avant sa mort le 19 février 1986 à Paris : « J'ai pour le Japon une très grande affection. J'ai vécu là-bas très près du peuple, des paysans, et j'ai beaucoup d'admiration pour les Japonais. Il y a chez eux des hommes qui savent unir de façon étroite l'esthétique et la vie quotidienne $»^{5}$. 

Damien Kunik de l'Université de Genève. Il m'écrit: "Depuis un certain temps, j'envisage de publier quelque chose au sujet d'un chapitre très spécifique de l'ouvrage Le Geste et la Parole intitulé "L'esthétique fonctionnelle ». Or, il m'apparaît aujourd'hui que ce chapitre n'aurait pu exister si Leroi-Gourhan n'avait pas séjourné longuement au Japon et n'avait pas fréquenté un groupe d'intellectuels et d'artisans préoccupés par la théorisation de la " beauté de la fonction ". Je suis moi-même très attaché à l'œuvre théorique de ce groupe et m'intéresse de fait à l'influence qu'il a eue sur une des grandes personnalités de l'ethnologie française. La thématique serait aussi une opportunité de révéler des jeux d'influences méconnus entre France et Japon ». Il ajoute : «Ce qui est réellement nouveau, par rapport à ce qui a été écrit jusqu'ici sur Leroi-Gourhan et le Japon, c'est que mes collègues et moi-même avons récemment retrouvé l'ensemble de ses archives japonaises. Celles-ci, versées depuis à Paris $\mathrm{X}$ et à l'EFEO, permettent de comprendre beaucoup plus précisément les relations de LeroiGourhan et de ses interlocuteurs japonais durant le séjour de 1937-1939. »

André-Georges Haudricourt prisait, lui aussi, les techniques et les hommes. J'ai eu la chance de le rencontrer et de l'accompagner lors de séjours en Bretagne chez Annick Levy-Ward à Erquy. Lorsqu'il se rend au Japon à l'invitation de son étudiant japonais, rien n'est laissé à l'improvisation, ni à l'imprévu. Le voyage a été soigneusement préparé et les deux protagonistes ont échangé à propos du programme par courrier avant le séjour.

Dans ce que Tadahiko Shintani décrit du séjour d'Haudricourt du 2 au 24 octobre 1978, je reconnais l'image qu'il me reste de lui. D'emblée et comme le titre l'annonce : « Dans l'intimité du professeur A.-G. Haudricourt - son voyage au Japon », le récit se donne dans la précision des faits et gestes d'Haudricourt : ce qu'il observe, ce qu'il mange, comment il marche, ce qu'il lit, de quoi il parle, ce qu'il exprime, ce qu'il dit, ce qu'il ressent. Shintani note qu'Haudricourt se montre quelquefois " irrité », qu' « il n'a pas caché son mécontentement ", et aussi qu'" il avait l'air heureux ». Et quand on lui demande ce qu'il pense du Japon ou plutôt «comment il avait trouvé le Japon?", Haudricourt répond : «Le Japon est un pays terrible : il n'y a pas de forêt naturelle. Partout ce sont des sugi (Cryptomeria japonica) plantés!».

15 Ce qui ne laisse de surprendre le lecteur de ce court récit, c'est l'absence d'idée quelque peu générale qui nous apprendrait ce que ce voyage a fini par imprimer chez Haudricourt. Comment Haudricourt a été affecté par son voyage au Japon? C'est tout au long de ce numéro du Portique, le fil rouge de notre préoccupation. Néanmoins, on découvre, et c'est par le truchement de son hôte, un Haudricourt "conférencier ", "amateur de lecture", "amoureux de Wajima», "adorateur de la Nature», « infatigable » et non moins énigmatique, et aussi et sans doute « impressionné ».

Une première remarque me vient à la lecture du texte de Shintani : en livrant ses observations, l'hôte japonais se laisse conduire par les effets que produit sur lui la présence d'Haudricourt sur ses terres natales. Il n'occulte tout à la fois ni le plaisir de la rencontre, ni la connaissance, qui peut être tirée de l'expérience de la rencontre. Il ne les sépare pas de la nostalgie, encore moins de la reconnaissance mutuelle. Le récit d'une journée particulière à Wajima - le pays natal de Shintani - nous permet d'illustrer cette idée. Shintani forme le projet d'emmener Haudricourt à Wajima ; il s'enquiert au préalable (on imagine par courrier ou au téléphone) de l'approbation d'Haudricourt. Puis c'est la réalisation du voyage et la journée entière passée à 
« herboriser » dans le jardin en friche autour de la maison à Wajima. Lorsque Shintani revient quelque temps après à Paris, Haudricourt lui présente " une lettre d'un missionnaire catholique qui visita Wajima il y a une centaine d'années, parue dans les Annales de l'Extrême Orient (Vol. 5. 1883) ». Haudricourt pointe ensuite, sur une carte de France, le village d'Haudricourt en disant « Voilà mon Wajima, c'est ici ». Ce qui fait dire à Shintani qu'Haudricourt a encore beaucoup de choses à lui apprendre.

Ma vie dans une campagne bretonne, entre Rennes et Dinan, jusqu'au milieu des années 70 , écrire m'a sans doute donné un certain goût pour les « choses » : en vrac, le travail artisanal, les plantes cultivées, les modes de construction, la collecte et la pêche. Plus tard, circonstances et formation universitaire ont fortifié ce goût. Je rencontre Haudricourt en 1989 et j'effectue un stage au Département de technologie comparée (Musée de l'Homme) dirigé alors par Françoise Cousin où Hélène Balfet collaboratrice de Leroi-Gourhan y avait encore des habitudes de travail. En balade à travers la Bretagne, A.-G. Haudricourt n'oubliait pas de m'enseigner l'origine des techniques et de me livrer aussi quelques souvenirs. Il m'apprit ainsi qu'il s'était rendu au Japon, et me parlait des Japonais comme d'« un peuple de pirates ». J'avais été si surprise par l'expression «pirates » que je ne lui ai pas même demandé ce qu'il entendait par là. Il me disait aussi combien nous étions pétris de tabous, de toutes ces idées qui nous empêchent de penser, de comprendre et de parfaire nos connaissances.

Alors que je relis la description de son voyage au Japon, me revient en mémoire l'esprit malicieux d'Haudricourt à vouloir surprendre ou éprouver son auditeur. Je comprends bien qu'Haudricourt n'avait pas voulu blesser ses hôtes en qualifiant le Japon de pays terrible où il n'y avait pas de forêt naturelle, ni, devant moi, mépriser les Japonais en les affublant de ce qualificatif de " pirates ». Haudricourt au style direct, sans ambiguïté, n'avait pas trouvé d'expressions plus synthétiques pour dire ce qu'il pensait du Japonais. Il suffit d'ôter la connotation péjorative de l'emploi du mot pirate, " individu (sans scrupules) qui s'enrichit (aux dépens) d'autres », pour deviner sa pensée. Disant que les forêts n'y sont pas naturelles, Haudricourt insistait sur l'absence d'une grande diversité des espèces botaniques et de l'omniprésence d'une espèce endémique. À force d'interventions, les Japonais auraient réduit la diversité des espèces végétales, insinue-t-il en bougonnant: « Il ne faut pas nettoyer comme ça. Il faut quand même laisser quelques feuilles pour les botanistes » et «Il ne faut pas enlever toute l'herbe comme ça. Il faut laisser de l'herbe pour les daims. Ah, pauvres daims! Comment peuvent-ils se nourrir comme ça!».

19 Haudricourt, reconnu pour sa science du concret, n'ignorait pas pour autant la présence des sentiments et le jeu des affects. Son court (et remarquable) essai Domestication des animaux, culture des plantes et traitement d'autrui, nous en donne la preuve dès les premières lignes : «Vis-à-vis du monde végétal et animal, à partir du néolithique, l'homme n'est plus seulement un prédateur et un consommateur, désormais il assiste, il protège, il coexiste longuement avec les espèces qu'il a domestiquées. De nouveaux rapports se sont établis, d'un type "amical", et qui ne sont pas sans rappeler ceux que les hommes entretiennent entre eux à l'intérieur d'un groupe $» .^{6}$

Une note publiée le 15/02/2019 par l'AFP informe : « Le gouvernement japonais a présenté vendredi un projet de loi pour reconnaitre les Aïnous en tant que peuple "indigène" de l'archipel, après des décennies de discrimination. Les Aïnous, dont de nombreux vivent dans la région de Hokkaido (nord), ont longtemps subi une politique 
d'assimilation et d'oppression. Leur langue a été interdite à la fin du $19^{\mathrm{e}}$ siècle et ce jusqu'en 1997, et leurs coutumes longtemps bannies. "Il est important de promouvoir l'honneur et la dignité du peuple aïnou et de les transmettre à la prochaine génération", a déclaré le porte-parole de l'exécutif, Yoshihide Suga, devant la presse. Cette loi, la première du genre, vise à l'instauration de mesures pour soutenir les communautés, doper l'économie locale et le tourisme. Elle doit être votée au cours de la session parlementaire actuelle, qui s'achève le 26 juin. "

Les Aïnous, nous les rencontrons dans une communication de Leroi- Gourhan au Japon, où il est déjà question d'une forme de tourisme qui aurait dévoyé ces populations. $\mathrm{Ce}$ qui entraîne le reproche de manquer d'authenticité. Elles sont en quelque sorte présentées comme perdues pour l'ethnologie. Nonobstant ce contexte difficile, LeroiGourhan s'approchera des Aïnous, rencontrera l'archéologue anglais Munro qui leur est resté attaché. Il écrit : « Les Aïnous m'intéressaient depuis 1934 comme l'un des peuples les plus singuliers du Pacifique nord (...). Ce que nous avons vu [son épouse et lui] a dépassé notre pessimisme préconçu. Il y a encore quelques villages à Karafuto où les débris de quelques tribus puissantes végètent (...) les dernières familles ont remonté la rivière et vivent autour du vieux Munro, l'archéologue anglais qui écrivit il y a trente ans un livre qui est resté fondamental sur l'archéologie du Japon et qui vit, ou plutôt vit peut-être encore car je l'ai quitté très malade, dans le dernier noyau des Aïnous de Nieptani. $»^{7}$

"Quel bilan? » s'interroge le biographe de Leroi-Gourhan, Philippe Soulier : « s'il faut tirer un bilan de son expérience japonaise, il est possible de répondre, au vu de tous ces projets non aboutis, que celui-ci peut sembler anecdotique et faible (...). Il ajoute: " Cette expérience s'avère forte dans la formation de sa pensée en ethnologie et sur la place de l'analyse technique des objets ». Il conclut: «l'expérience japonaise participe donc directement à sa formation épistémologique, ouverte sur les interactions et les limites entre champs d'étude». Si explicite soit cette réponse, je continue à m'interroger sur les conditions de recueil de l'information, les contextes d'obtention des données, ses interlocuteurs institutionnels mais aussi et surtout les informateurs rencontrés, les relations qui se nouent lors de ce séjour de Leroi-Gourhan. Ces questions me semblent décisives, elles participent d'une réflexion à avoir sur " la relation, si angoissante, avec "le terrain" " ainsi qualifiée par Pierre Bourdieu dans la préface d'Un ethnologue au Maroc de Paul Rabinow ${ }^{8}$, qui poursuit: "Qu'est-ce qu'un informateur et que fait-il exactement lorsqu'il élabore, à l'intention de l'anthropologue, une représentation de son propre monde, dont on ne sait jamais clairement si les schèmes selon lesquels elle est informée, mise en forme, sont empruntés au système des structures cognitives caractéristiques de sa propre tradition ou au système de l'ethnologue, ou à un mixte, inconsciemment négocié, des deux codes collectifs de classification qui se trouvent confrontés? ».

En 1993, au cours d'un entretien pour la télévision japonaise, et sans doute pour les besoins d'une émission publique Junzo Kawada pose à nouveau à Lévi-Strauss les questions de l'« intérêt anthropologique » et de « l'attachement pour le Japon ». Lequel répond : " Je ne dirais pas que l'intérêt était continu. Pendant mes années au Brésil, j'étais entièrement envahi par les choses de l'Amérique et je ne pensais plus beaucoup au Japon. Mais même aux États-Unis pendant la guerre je recommençai à m'y intéresser très vivement, en regardant les objets dans les musées... Et, au fond, je ne pensais pas que j'irais jamais au Japon. C'était une idée qui ne m'était pas venue. Puis une invitation 
merveilleuse de la Fondation du Japon en 1977 est arrivée, presque comme une sorte de coup de tonnerre. Et je me suis dit : mais enfin je vais voir le Japon auquel, par intermittences, j'ai pensé toute ma vie ».

Lévi-Strauss se voit offrir un cadeau : l'invitation rejoint un désir longtemps caressé. Leroi-Gourhan quant à lui reconnait sans doute dans ce voyage le cadeau de mariage d'un de ses professeurs qui participait au jury d'élection des boursiers pour le Japon. Le voyage de Haudricourt peut être vu comme une forme de reconnaissance d'un étudiant (devenu professeur d'université) à son professeur. Je n'ai pas interrogé les universitaires qui se rendent aujourd'hui au Japon, il me semble que ces voyages sont initiés à la demande des universités japonaises ou bien s'inscrivent dans des échanges entre universités. Ces invitations s'appréhendent dans une forme d'accomplissement de la politique d'ouverture du Japon initiée dès l'ère Meiji, une forme de diplomatie culturelle. Cette diplomatie culturelle, nous en parlons avec Clélia Zernik dans l'entretien qu'elle m'a accordé', notamment à propos des expositions qui ont eu lieu en France l'an dernier. Le dispositif d'échanges qui encadre ces voyages en détermine partiellement l'expérience. Jean-Luc Nancy décrit clairement dans Nippospitalité ${ }^{10}$ combien un « accident de la vie quotidienne » ouvre avec bonheur, pourrait-on dire, un espace qui échappe à la condition de touriste.

Dans la revue d'études japonaises, Ebisu, un article de Damien Kunik nous éclaire sur certaines rencontres que fait Leroi-Gourhan au Japon : « Il a ponctuellement fréquenté Yanagi Muneyoshi, chef de file du Mouvement des arts populaires et fréquenté régulièrement, avec une amitié affichée, Kawaï Kanjiro (1890-1966), céramiste et autre figure centrale des Arts populaires, intime de Yanagi ». À travers l'exploration de cette " posture » et de cette " sensibilité à laquelle l'initie le céramiste ", il resterait à viser " l'engagement » de Leroi-Gourhan au Japon.

Je relève de mon côté que Leroi-Gourhan reviendra dans Les racines du monde sur cette rencontre : "J'ai connu à Kyôto un certain nombre d'artisans, et, en particulier, un potier célèbre, Kawaï Kanjiro. (...) J'allais chez Kawaï pour le voir travailler, ou pour discuter avec lui. La technique m'intéressait depuis longtemps, ce doit être une chose profondément inscrite en moi, mais l'abord matériel des objets, le travail, la pensée d'un artisan ne me sont devenus perméables qu'à partir de mon voyage au Japon ».

Quand Leroi-Gourhan parle de ce "travail d'observation » de l'artisan, on devine ses attentes, ses aspirations et la considération qu'il lui porte, lequel est devenu un informateur dans le sens entendu en ethnologie. A-t-il conscience pour autant qu'il vient de rompre avec une image de l'explorateur encore bien active à cette époque (qu'on pense à la Croisière Jaune qui démarre en 1931 en pleine effervescence coloniale et dont le chef de l'expédition, Georges-Marie Haardt meurt à Hong Kong en 1932).

28 Je voudrais attirer l'attention sur la relation si particulière qui se noue dans l'observation d'un geste, d'une intention et d'une réalisation techniques, de «l'ensemble de procédés employés pour produire une œuvre ou obtenir un résultat déterminé ». Cette proximité nécessaire, et accomplie pour les besoins d'une enquête ethnologique, est exigeante ; elle requiert beaucoup de tact parce qu'elle est contenue dans une opération délicate de transfert, de transmission, d'enseignement... elle est évidemment affectée. Quand Damien Kunik parle d'amitié à l'endroit de ces relations que noue Leroi-Gourhan avec des artisans, il circonscrit une catégorie à ajouter à la méthodologie ethnologique. 
non l'absence d'interactions. Il y en aura, bien sûr, mais rien ne me semble de l'ordre de ce que j'appelle la rencontre, sauf peut-être le tout dernier soir quand Ryotaro, un jeune danseur de Kabuki surgit dans notre logement et tourbillonne d'une joie non feinte. Didier l'avait rencontré lors de son premier séjour (il lui avait simplement demandé son chemin dans la rue). Et ce soir-là, il est venu rencontrer Didier à nouveau et lui offrir, à la japonaise, un présent. Dans son enthousiasme, il nous fait une démonstration en kimono de travail de son art. Il est déjà prêt, nous dit-il, à venir danser à Paris. Il est originaire de l'île d'Hokkaïdo où vit sa mère.

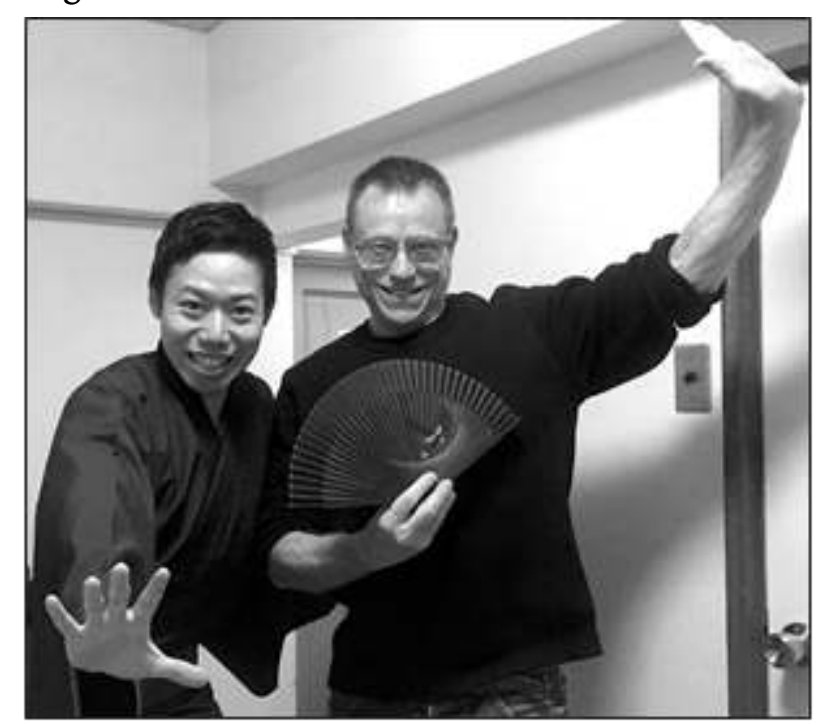

33

J'entends à la radio, quelques mois après mon retour : « Au Japon, un employé d'accueil du parc Shinjuku Gyoen à Tokyo a laissé entrer gratuitement les touristes étrangers -par peur d'interagir avec eux- pendant une période de deux ans et demi ».

34 Aurais-je relevé cette information si je n'avais pas autant aimé me promener dans les jardins japonais? Y observer le travail des jardiniers, méticuleux voire maniaque, y compris dans les jardins secs ; m'arrêter devant les photographes amateurs qui 
attendent patiemment le passage d'une espèce d'oiseau ; relever le tuteurage appliqué sur des vieux arbres tout rabougris, le nouage des bambous pour la confection de clôture ou de haies, les jeux de fermeture et d'ouverture des portails, la disposition des pierres levées et les pas japonais, etc. Autant d'occasions pour moi de me réjouir et d'entrer dans une sorte d'intimité, l'intimité des gestes du soin, auxquels m'avaient sensibilisée certains jardiniers qui venaient entretenir le potager de la maison familiale en Bretagne, et qu'Haudricourt avait comme magnifiée dans certains de ses articles et prolongée dans la pratique d'herboriser (bien qu'elle ne soit pas du soin, à proprement parlé, puisqu'elle consiste à découvrir la présence et les noms de ces plantes que nous rencontrons sur notre chemin). Parfois il nous arrivait que nous partions à la découverte des plantes, «nouer une amitié » avec elles, en nous arrêtant sur les bords de route ou aux bords des côtes.

Prendre la file dans les musées, adopter le rythme des visiteurs la plupart japonais, c'est-à-dire lent, avancer très lentement dans la pénombre du Musée d'art Nezu, scruter une pièce, retenir son souffle en relevant la datation, admirer, s'étonner. La visite des musées est active et j'en sors souvent hébétée. Le seul avantage est que mes rêves en seront que plus riches.

Du quai de la gare, où nous attendons la rame qui nous ramènerait à notre quartier, nous avons repéré ce restaurant en contrebas. Il nous parait différent de ceux que nous avions jusqu'alors fréquentés. Nous distinguons de l'extérieur l'animation intérieure et son allure confortable. Dès que nous entrons, s'impose la présence du chef-cuisinier qui se tient debout, avec son air impassible, ses lunettes rondes et sa minuscule moustache, il me fait penser à un portrait de Foujita. Il se tient un peu en hauteur, au milieu d'un espace dédié au travail qui est à la fois coupé du reste du restaurant tout en communiquant avec lui. Un bar d'une bonne hauteur court tout autour. Des clients peuvent s'asseoir et s'y attabler, les yeux sur le travail du chef et de ses deux adjoints qui se tiennent un peu en retrait. Une fois installés en contre bas de l'espace de travail, nous consultons le menu, nous commandons et nous verrons arriver les plats les uns après les autres. Cette première fois, nous sommes situés sur un côté de l'espace de travail, c'est un adjoint qui prend la commande, nous comprenons que nous ne devons pas nous attarder étant-donné l'heure avancée. Nous sommes complètement ravis des mets servis au point que nous nous promettons de revenir.

C'est ce que nous faisons quelques jours après. Nous n'arriverons pas si tard pour avoir suffisamment de temps et profiter du moment. Nous avons été placés cette fois face au chef -qui avait remarqué notre présence mais sans paraitre nous prêter quelque attention. Il nous invite d'un simple regard à nous placer quasiment en face de lui. Nous tournons le dos à la porte d'entrée du restaurant. Une vitre nous sépare de l'espace du travail. Cette situation de proximité nous met dans une contrainte, celle de porter notre attention au chef-cuisinier. Dans un premier temps, nous sommes un peu gênés mais le plaisir prend le dessus, en tout cas pour moi, d'observer le travail. Les gestes sont tout-à-fait remarquables ; ils semblent solliciter uniquement les mains, le buste restant parfaitement droit. Les mouvements, restreints, sont accomplis dans une économie d'espace. Il n'y a aucune gesticulation, aucune amplitude, l'œil semble commander les opérations et notamment les mains. La bonhommie qu'on prête volontiers aux cuisiniers, la générosité que nous leur connaissons, sont ici comme envolées ; on est aimanté par des gestes rapides, sûrs, et précis. Ce restaurant s'est spécialisé dans les yakitori ; il y a bien d'autres plats à la carte mais les brochettes sont 
ce que nous avons choisies et le chef aux commandes les prépare sans faillir. J'ai aussi relevé l'attention qu'il portait aux faits et gestes d'une jeune femme apparemment en apprentissage ; les échanges entre le chef et l'apprentie sont uniquement portés par la nécessité de l'apprendre et du transmettre.

Cette configuration en présence - le client assistant à la préparation des mets - n'est pas rare. On la retrouve dans bien des restaurants au Japon et aussi dans certains restaurants japonais en France. C'est comme la signature d'un genre. À Nara, sur le chemin du retour vers la gare, cherchant un restaurant, nous nous sommes laissés guider par une vieille femme qui nous a menés jusqu'à une échoppe nichée au fond d'une galerie : elle nous a installés et confiés aux bons soins d'un couple, le chef et sa femme. Lui est un homme plutôt âgé, il se tient devant nous, préparant des tempura de différentes sortes, fritures embrochées sur des baguettes de bois, d'asperges et même de camembert, qu'il nous remet une à une en nous indiquant l'assaisonnement qu'il convient de choisir. Là aussi, les gestes sont modestes, retenus, au sens d'une politesse et d'une délicatesse, dédiés à l'art de l'accommodement du mets mais aussi des hôtes que nous sommes. Nous ne sommes pas les seuls à nous voir accorder ce privilège. La dame-guide revient vers nous pour s'assurer de notre satisfaction. Nous sommes bien embêtés, ne sachant pas comment la remercier. Elle nous salue plusieurs fois en repartant, nous laissant entre les mains du cuisinier à qui nous formulons nos compliments. Il nous adresse via son smartphone une formule en anglais disant qu'il est content que nous soyons contents.

Ce soir-là, comme l'autre soir à Tokyo, j'ai perçu dans le geste du cuisinier, un rythme, des manières de se mouvoir, des attentions, des soins. J'ai repensé à ces jardiniers que j'avais repérés dans les différents jardins, à Kyoto et Tokyo. Leurs présences et leurs gestes sont, ici encore, éminents. Leur travail est aussi minutieux que celui de retirer des branches de pins les aiguilles fanées ou roussies, par exemple. Les jardiniers manipulent sans bruit des petits outils fixés près du corps, dans une grande économie de gestes. Les végétaux, surtout les arbres, sont traités individuellement, les tuteurs en témoignent, comme les noms qui leur sont donnés. D'où vraisemblablement leur longévité, et peut-être aussi l'attachement affectueux qu'ils finissent par susciter ${ }^{12}$. À moins d'inverser le raisonnement : en leur donnant un nom, on les place dans une proximité affectueuse, on en prend soin.

Une manière d'accueillir et de recevoir, d'étendre aux plantes ou aux mets l'amitié qui nourrit et façonne aussi la connaissance, d'appliquer la lenteur à l'observation, seraient-ils les moyens d'atteindre à l'autre dont le processus est si fascinant notamment au Japon? 


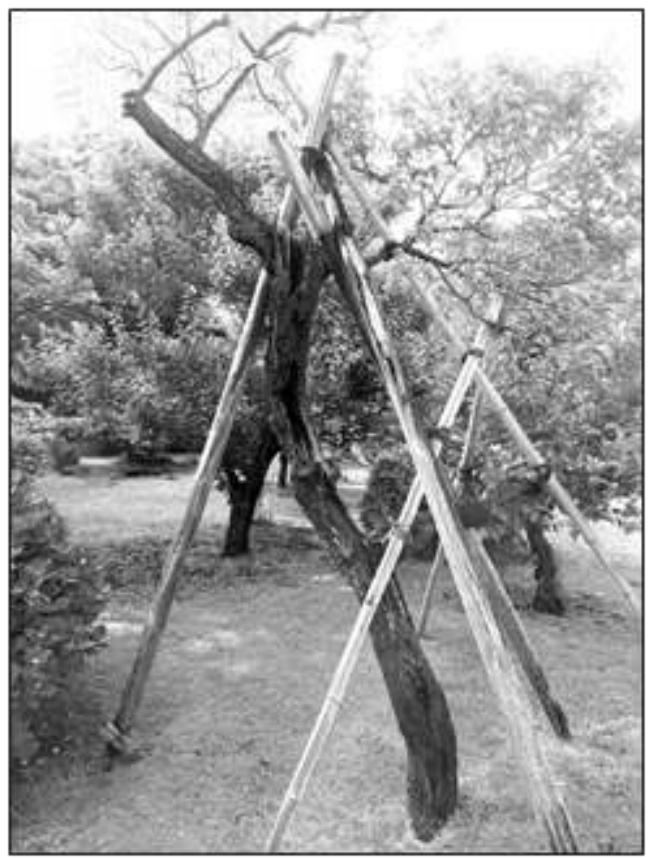

Arbre tuteuré, Jardin Hamarikyu, Tokyo

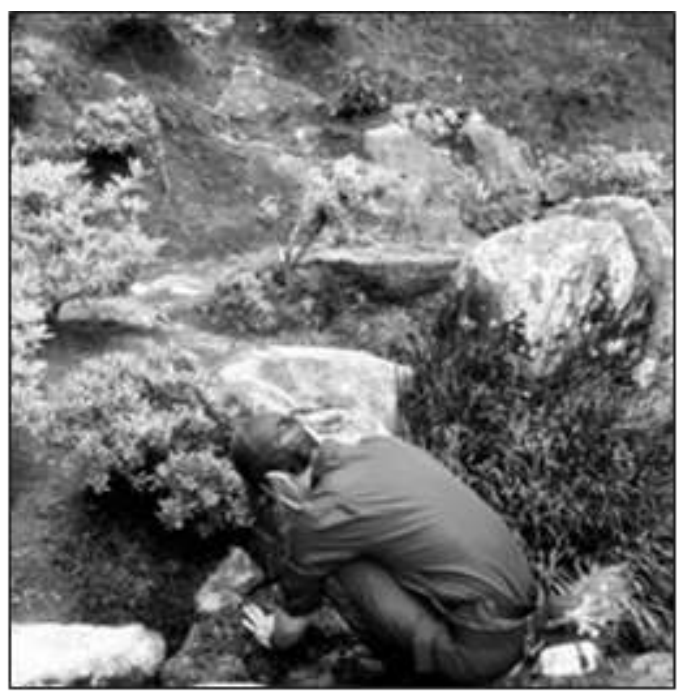

Jardinier, Ginkaku-ji, pavillon d'argent, Kyoto 


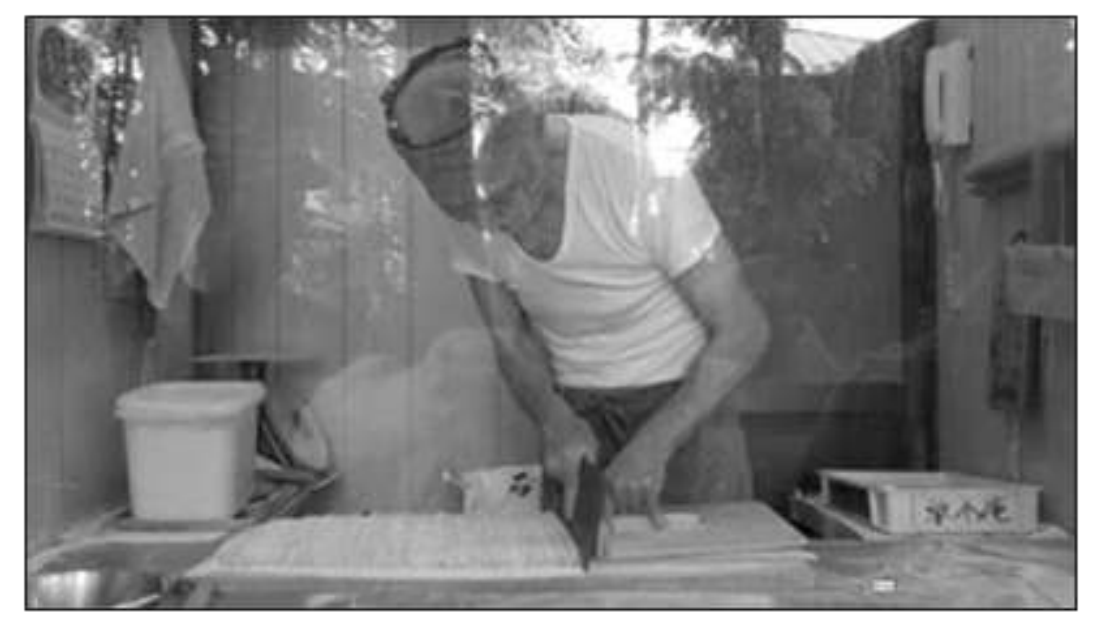

Découpe de pâtes udon, Tokyo

\section{NOTES}

1. Cf. supra Philippe Choulet, « Lévi-Strauss et le Japon : l'éthos de la distance ».

2. Cf. Noel Barbe, Jean-François Bert, (ss. dir), Penser le concret, Creaphis éditions, Paris, 2011.

3. Michel Naepels, "Une étrange étrangeté. Remarques sur la situation ethnographique ", L'Homme, 1998.

4. Frédéric Joulian, « Du Japon et d'ailleurs », Techniques et Culture, n57, 2 à 11/2, pp. 6-13.

5. André Leroi-Gourhan, Les racines du monde. Entretiens avec Claude-Henri Rocquet, Belfond, Paris, 1982.

6. A.-G. Haudricourt, « Domestication des animaux, culture des plantes et traitement d'autrui », L'Homme, 2(1), 1962.

7. André Leroi-Gourhan, « Conférence du 15 janvier 1940 à l'Institut Français d'Anthropologie », Pages oubliées sur le Japon, op.cit., p. 414.

8. Paul Rabinow, Un ethnologue au Maroc. Réflexion sur une enquête de terrain (1977), préface de Pierre Bourdieu, Hachette, coll. Histoire des gens, 1988 pour la traduction française.

9. Cf. Infra, « Les nouvelles figures de l'art contemporain japonais. Entretien avec Clélia Zernik ». 10. Cf. Supra, Jean-Luc Nancy, « Nippospitalité».

11. Clifford Geertz, Ici et Là-Bas. L'anthropologue comme auteur (1988), traduit de l'anglais (USA) par Daniel Lemoine, Éditions Métailié, Paris, 1996, p. 144. 


\section{RÉSUMÉS}

Le récit d'un séjour, au printemps 2018, à des fins touristiques, mêle souvenirs et retours d'expériences. Les deux ethnologues A.-G. Haudricourt et André Leroi- Gourhan accompagnent la réflexion sur les conditions et les possibilités de la rencontre avec l'autre.

The story of my stay for tourist purposes in Japan, realized in May 2018, mixes memories and feedback. The two ethnologists A.-G. Haudricourt and André Leroi- Gourhan who stayed in Japan for three weeks in 1978 for the first; almost two years from 1937 to 1939 for the secondaccompany my reflection on the conditions and possibilities of the meeting with the other.

\section{AUTEUR}

\section{CATHERINE SIMON}

Catherine Simon, titulaire d'un D.E.A. Histoire des techniques (EHESS, Paris), a effectué plusieurs enquêtes dans l'Est et l'Ouest de la France mettant en jeu des sociétés locales et des aspects de leur environnement naturel (l'industrie granitière dans les Vosges, des carrières de pierre de taille dans la Meuse, les salines de Dieuze, les femmes dans la vallée sidérurgique de la Fensch, la culture du blé- sarrasin en Bretagne, etc.). Depuis 2003, elle anime le Forum-IRTS de Lorraine (site de Ban Saint-Martin). 